\title{
Systemic Amyloidosis as a Rare Complication of IgG4 Related Disease: Case Report and Literature Review
}

Leonardo Oliveira Mendonca ( $\square$ leonardo.oliveira.mendonca@gmail.com )

Universidade de Sao Paulo Faculdade de Medicina https://orcid.org/0000-0001-7533-1937

Henrikki Gomes Antila

Universidade de Sao Paulo Faculdade de Medicina

Alex Isidoro Prado

Universidade de Sao Paulo Faculdade de Medicina

Luiz Augusto Marcondes Fonseca

Universidade de Sao Paulo Faculdade de Medicina

Miton de Arruda Martins

Universidade de Sao Paulo Faculdade de Medicina

Rafael Dyer

Universidade de Sao Paulo Faculdade de Medicina

Jorge Kalil

Universidade de Sao Paulo Faculdade de Medicina

Fabio Fernandes Morato-Castro

Universidade de Sao Paulo Faculdade de Medicina

Myrthes Anna Maragna Toledo-Barros

Universidade de Sao Paulo Faculdade de Medicina

\section{Research Article}

Keywords: Immunoglobulin 4 Related Disease (IgG4-RD), Systemic Amyloidosis, rare complication, disease, case report

Posted Date: July 16th, 2021

DOI: https://doi.org/10.21203/rs.3.rs-519892/v1

License: (1) (1) This work is licensed under a Creative Commons Attribution 4.0 International License. Read Full License 


\section{Abstract}

Immunoglobulin 4 Related Disease (IgG4-RD) is immune-mediated fibroinflammatory disease and despite recent advances the immunological process involved in the disease pathogenesis is still unclear. Serum amyloid $A$ (SAA) the precursor protein in AA amyloidosis is induced by inflammatory mediators such as IL-1, IL- 6 and TNF cytokines. The treatment of AA amyloidosis is directed by the theoretical cytokine involved in the underlying inflammatory condition. Many inflammatory conditions has already been associated to AA amyloidosis and secondary to IgG4-RD seems to be rare. Here we report the case of a Brazilian patient with IgG4-RD with a fatal evolution of systemic amyloidosis. We also revised the cases already reporte in the literature with IgG4-RD and systemic amyloidosis.

\section{Introduction}

Immunoglobulin G4 - related disease (IgG4-RD) is an uncommon systemic inflammatory condition mediated by infiltration of IgG4-producing plasma cells (1). Definitive diagnosis is stablished in biopsies of affected tissues with proven and variable intensity of IgG4 positive plasma cells infiltration. Up to $50 \%$ of patients do not have increased serum IgG4 levels 2 . Serum IgG4-plasmablast is the gold standard marker for diagnosis and disease remission. Rituximab is the gold standard in order to achieve clinical control, reducing progression of fibrosis and decreasing inflammatory markers $(1,2)$.

Amyloidosis comprises a group of diseases that are the product of the deposition of unfolded amyloid protein in tissues. In amyloid A amyloidosis (AA amyloidosis), pro-inflammatory cytokines such as interleukin 1, interleukin 6 and TNF-a seems to interact synergistically for the serum amyloid A proteins (SSA) synthesis in the liver, which can be deposited in various organs leading to tissue damage. Prolonged or recurrent episodes of systemic inflammation, as observed in systemic autoimmune, autoinflammatory or infectious disease, is the main cause of AA amyloidosis. Unexpectedly, systemic AA amyloidosis has no specific treatment and it is recommended to block the likely underlying cytokine involved in the process (3).

Amyloidosis is a rare complication of IgG4-RD with few cases already reported. The immunology process involved in amyloid formation in patients with IgG4-RD is not yet elucidated neither the phenotypes more susceptible to systemic amyloidosis. This paper aims to report the case of a Brazilian patient with IgG4$\mathrm{RD}$ refractory to treatment who has evolved dramatically to systemic amyloidosis, and to review the cases already reported in the literature.

\section{Material And Methods}

We retrieved patient's data from clinical records. We performed a literature review of cases already reported with both IgG4-RD and systemic amyloidosis.

\section{Case Report}


A 37-year-old black male patient, previously healthy, came to our outpatient clinic with a nine-year history of persistent night fever and night sweating, associated with pain in the right hypochondrium radiating to the epigastrium. The patient also presented iron deficiency anemia refractory to iron replacement, high platelet counts and positive inflammatory markers (CRP and ESR).

Initially all infectious, autoimmune and neoplastic known causes were excluded. An abdominal CT revealed hepatosplenomegaly and retroperitoneal mass. High-dose corticosteroids prescribed empirically at high doses were only partially effective.Histopathological analysis of retroperitoneal mass was compatible with IgG4-RD. Steroid-sparing agents were not effective in controlling symptoms, as well as tapering steroids dosage was also not able to reduce the levels of inflammatory markers. For this reason, B cell depletion therapy was initiated (ritutximab 1g/dose every 4 weeks in two doses). Clinical control could be observed but without considerable reduction in inflammatory markers.

Due to the socioeconomic context, the patient had low adherence to the proposed treatments and to following the check-up routine.

Six years after diagnosis, he was admitted to the emergency room with severe right hypochondrium pain, after presenting hematochezia for twenty days.

Laboratory findings included a significant increase in inflammatory markers, hypogammaglobulinemia, hypoalbuminemia, high pro-BNP levels and nephrotic proteinuria with normal renal function. Imaging exams showed increased hepatosplenomegaly (Fig. 1). Also, hemorrhagic gastroduodenitis was diagnosed during an upper gastrointestinal endoscopy. Multiple specimen biopsies were obtained from esophagus, stomach, duodenum and liver. Amorphous material deposits were identified in all biopsy tissue samples, with a positive Congo red stain (Fig. 1). Subsequently, it was observed a serum amyloid A protein of $186 \mathrm{mg} / \mathrm{L}$ (reference value below $6.4 \mathrm{mg} / \mathrm{L}$ ). Empirical treatment with colchicine was initiated and anti-IL6 was proposed. However, after two months of hospital discharge, and before anti-IL 6 could be started, he developed septic shock refractory to clinical measures progressing to death. All relevant laboratory data and treatment used are shown in Fig. 1.

\section{Discussion}

We described the case of a patient with recalcitrant IgG4-RD with persistently high inflammatory biomarkers and a sudden worsening of his clinical condition, leading to the diagnosis of AA systemic amyloidosis.

The immunopathogenesis of IgG4-RD interestingly provokes mechanist insights into systemic inflammation that can lead to amyloid formation once lgG4 has an anti-inflammatory property rather compared to other immunoglobulin $\mathrm{G}$ sybtypes. Cytokines, that are key pieces for amyloid formation also accounts for IgG4 related disease. 
Cytokines derived from innate immune system seems to be involved in the pathogenesis of IgG4-RD. From one side IL-6 seems to be associated to vascular forms of IgG4-RD whereas Type I IFN, specially Type I IFN-a, was related to IgG4-RD pancreatitis. Also in vitro studies revealed that IgG4 class-switch DNA recombination is enhanced in the presence of IL -4 and BAFF (B cell activation factor) or APRIL (proliferation-inducing ligand), which strongly suggests that BAFF and APRIL are involved in B cell survival as well as in IgG4 production.

Several reports suggests that IL-6 does not play a major role in the inflammatory responses in IgG4-RD. As IgG4-RD is characterized by Th2 rather than Th1 cytokines, differentiation and activation of M2 macrophages is more likely to be induced in this disease.Thus, M2 macrophages can contribute to tissue fibrosis via IL-10 and IL-13 production in IgG4-RD (4).

Amyloidosis following IgG4-RD is a rare complication. We identified six cases of IgG4-RD and amyloidosis in the literature, all summarized in Table 1 . All patients were adult and $66 \%$ were female. There was no specific subtype of IgG4-RD more likely to be involved in amyloidosis formation. Time from the IgG4-RD presentation to the diagnosis of amyloidosis vary in cases reported from 5 months to 20 years and mass spectrometry was performed in just one case to subtype the amyloid protein. Successful treatment after amyloidosis was installed could be obtained using high doses of systemic steroids and anti-CD20 monoclonal antibodies. Two cases with localized breast Rosai-Dorfman IgG4-RD were successfully treated with tumor resection and both reported to be disease free after a long follow-up.

Based on this case series herein reported, we strongly suggests systematic screening of this complication on follow-up of patients with IgG4-RD, specially for those who: 1 - don't achieve disease's control with the usual theraphy; and/or 2 - have persistent high levels of inflammatory markers. We also raise discussions on the physiopatology of SAA formation in patients with IgG4-RD.

\section{Declarations}

\section{Funding:}

There was no funding supporting this paper

\section{Conflicts of interest/Competing interests:}

All authors declare no conflicts/competing interests related to this paper

\section{Ethics approval:}

this paper was approved by the Ethical Committee at the University of São Paulo

\section{Consent to participate:}

We obtained consent to participate previously of the patient died 


\section{Consent for publication:}

We obtained consent for publication of a next kin of the patient

\section{Availability of data and material:}

Data are available at any time

\section{Authors' contributions:}

Leonardo Oliveira Mendonca, Henrikki Gomes Antila, Alex Isidoro Prado: article draft, data collection, patient care

Rafael Dyer: article draft and pathological images acquisition

Luiz Augusto Marcondes Fonseca, Mílton de Arruda Martins, Jorge Kalil,Fábio Fernandes Morato Castro,Myrthes Anna Maragna Toledo Barros: patient care and senior supervison

\section{References}

1. Stone JH, Zen Y, Deshpande V. IgG4-Related Disease. N Engl J Med 2012;366:539-51.

2. Bledsoe JR, Della-torre E, Rovati L, Deshpande V. IgG4-related disease: review of the histopathologic features, differential diagnosis, and therapeutic approach. 2018:459-476.

3. Wechalekar AD, Gillmore JD, Hawkins PN. Systemic amyloidosis. Lancet. 2016;387(10038):26412654.

4. Maehara T. Nihon Rinsho Meneki Gakkai Kaishi. 2017;40(3):206-212. doi:10.2177/jsci.40.206

5. Karim F, Clahsen-van Groningen M, van Laar JAM. AA Amyloidosis and IgG4-Related Disease N Engl J Med 2017;376;62.

6. Liu M, Li X, Li Y, Wang Z, Cheng L, Song X, Wu Y. Rosai-Dorfman disease with features of IgG4-related disease in the breast: Cases report and literature review. Asian Pac J Allergy Immunol. 2018 Mar;36(1):51-57. doi: 10.12932/AP0862. PMID: 28577522.

7. Wisniowski-Yáñez A, Zavala-García G, Hernández-Molina G, González-Duarte A, Delgado-de la Mora J, Ángeles-Ángeles A, Martín-Nares E. Amyloid A amyloidosis secondary to immunoglobulin G4related disease. Rheumatology (Oxford). 2021 Mar 2;60(3):e97-e98. doi:

10.1093/rheumatology/keaa467. PMID: 33040142.

8. Ichikawa T, Nakao K, Hamasaki K, Ohkubo K, Toriyama K, Eguchi K. An autopsy case of acute pancreatitis with a high serum IgG4 complicated by amyloidosis and rheumatoid arthritis. World $\mathrm{J}$ Gastroenterol. 2005 Apr 7;11(13):2032-4. doi: 10.3748/wjg.v11.i13.2032. PMID: 15801001; PMCID: PMC4305732.

9. Chowsilpa S, Chaiyasate S, Wannasai K, Daroontum T. Localised nasopharyngeal amyloidosis: the importance of postoperative follow-up. BMJ Case Rep. 2018 Feb 14;2018:bcr2017222067. doi: 
10.1136/bcr-2017-222067. PMID: 29444790; PMCID: PMC5847832.

10. Karim F, Clahsen-van Groningen M, van Laar JA. AA Amyloidosis and IgG4-Related Disease. N Engl J Med. 2017 Feb 9;376(6):599-600. doi: 10.1056/NEJMc1614275. PMID: 28177871.

\section{Table}

Due to technical limitations, table 1 jpeg is only available as a download in the Supplemental Files section.

\section{Figures}
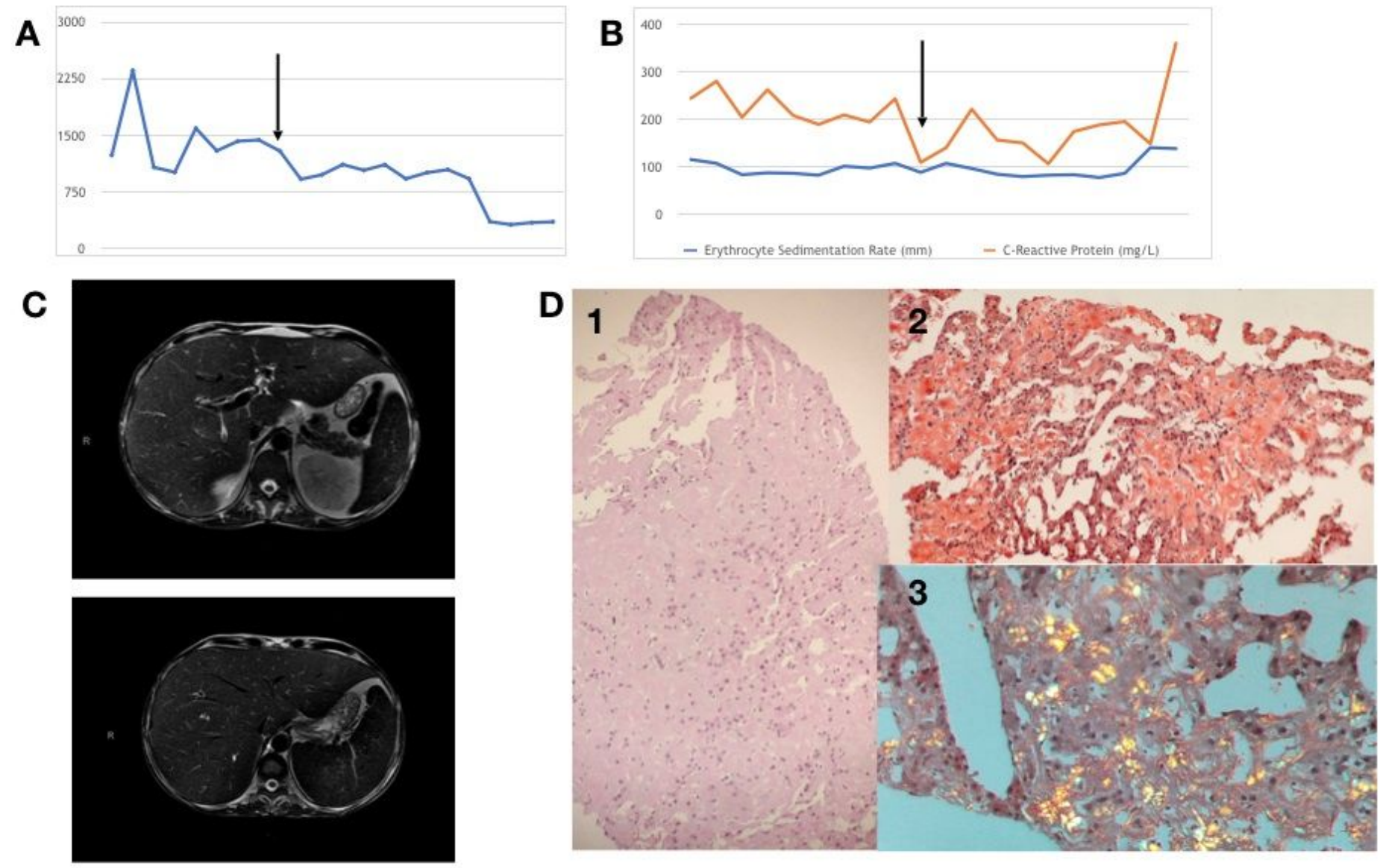

\section{Figure 1}

Laboratory analysis and histopahological findings consistent with systemic amyloidosis. A - High levels of immunoglobulin $\mathrm{G}(\mathrm{IgG})$ levels over the period of our observation with a consistent decrease in IgG levels after Rituximab (arrow). B - Inflammatory markers levels in the disease course, in orange CRP levels and in blue ESR levels and arrow indicates the first infusion of Rituximab. C - Liver radiological images obtained in the moment of AA amyloidosis was confirmed. D - Liver biopsy (Size: 1.6x1.4 cm) previously fixated under $10 \%$ neutral buffered formalin. 1 - Hematoxylin-eosin stains from the patient showing: 
Prominent sinusoidal ectasia with global amorphus deposit and diffusely altered architeture. 2-3 It is possible to aprecciate that after undergoing Congo red stain and polarized light, showing deposits of amyloid substance.

\section{Supplementary Files}

This is a list of supplementary files associated with this preprint. Click to download.

- FigureslgG4Amyloidosistocomprehensivemedicine.002.jpeg 